Homology, Homotopy and Applications, vol.13国, 2011, pp.329-335

\title{
MOTIVES OF SOME ACYCLIC VARIETIES
}

\author{
ARAVIND ASOK \\ (communicated by Charles Weibel)
}

\begin{abstract}
We prove that the Voevodsky motive with $\mathbb{Z}$-coefficients (resp. $\mathbb{Q}$-coefficients) of a $\mathbb{Z}$-acylic (resp. $\mathbb{Q}$-acyclic) smooth complex variety of dimension $\leqslant 2$ is isomorphic to that of a point and discuss some related extensions.
\end{abstract}

\section{Introduction}

A smooth connected complex variety $X$ such that $X(\mathbb{C})$, viewed as a complex manifold, has trivial reduced integral (resp. rational) singular homology will be called a $\mathbb{Z}$-acyclic (resp. $\mathbb{Q}$-acyclic) variety. The only $\mathbb{Z}$ - or $\mathbb{Q}$-acylic smooth variety of dimension 1 is $\mathbb{A}^{1}$. It is not immediately obvious that there exist $\mathbb{Q}$-acyclic varieties besides $\mathbb{A}^{n}$, but in fact the theory of such varieties is extremely rich; see Example 5 for a taste. We refer the reader to the excellent survey article [Zaí99] for many examples of $\mathbb{Q}$-acyclic varieties. The goal of this note is to study the motive, in the sense of Voevodsky, of such acyclic varieties, especially in the case where $\operatorname{dim} X=2$. In particular, our goal is to prove the following result:

Theorem 1. If $X$ is a $\mathbb{Z}$-acyclic (resp. $\mathbb{Q}$-acyclic) smooth complex variety of dimension 2 , then the canonical morphism $\mathbf{M}(X) \rightarrow \mathbb{Z}$ (resp. $\mathbf{M}(X) \rightarrow \mathbb{Q}$ ) is an isomorphism in $\mathbf{D M}_{g m}(\mathbb{C}, \mathbb{Z})\left(\right.$ resp. $\mathbf{D} \mathbf{M}_{g m}(\mathbb{C}, \mathbb{Q})$ ).

Assuming some "standard" conjectures regarding motives, a reformulation of the Hodge conjecture (see [Hub08, Proposition 3.4.1]) predicts that the Hodge realization functor, from Voevodsky's derived category of motives (with the Tate motive inverted) to an appropriate (derived) category of Hodge structures, is conservative. In particular, this conjecture implies that $\mathbb{Q}$-acyclic smooth complex varieties should have trivial rational motive. We can view the result above as providing a tiny amount of evidence for this conjecture. Given the known rationality results for $\mathbb{Q}$-acyclic varieties of dimension 2, the proof is quite easy. Our main hope is that Theorem 1 points to some interesting questions regarding the relationship between acyclic varieties and the theory of motives. For example, is it true that the integral Voevodsky motive of a $\mathbb{Z}$-acyclic variety is necessarily isomorphic to $\mathbb{Z}$ ?

Aravind Asok was partially supported by National Science Foundation Awards DMS-0900813 and DMS-0966589.

Received April 28, 2011, revised November 16, 2011; published on December 20, 2011.

2000 Mathematics Subject Classification: 14F42, 14R05, $19 \mathrm{E} 15$.

Key words and phrases: acylic, $\mathbb{A}^{1}$-homotopy, motive.

Article available at http://intlpress.com/HHA/v13/n2/a20 and doi:10.4310/HHA.2011.v13.n2.a20

Copyright (C) 2011, International Press. Permission to copy for private use granted. 


\section{Acknowledgements}

We thank Brent Doran for many useful discussions and the referee for suggestions to improve the exposition.

\section{Proof of Theorem 1}

For simplicity, we fix $\mathbb{C}$ as our base field.

\section{Triangulated categories of motives}

Regarding Voevodsky's derived category of motives, our terminology will follow Parts 4 and 5 of the book [MVW06]. Suppose $R$ is a commutative unital ring. Denote by $\mathbf{D M}_{-}^{\text {eff }}(\mathbb{C}, R)$ Voevodsky's derived category of effective motivic complexes with $R$-coefficients (see MVW06 Definition 14.1); we will only consider the cases $R=\mathbb{Z}$ or $\mathbb{Q}$ in the sequel. For a smooth scheme $X$, the motive of $X$ with either integral or rational coefficients is an object of $\mathbf{D M}_{-}^{\text {eff }}(\mathbb{C}, \mathbb{Z})$ or $\mathbf{D} \mathbf{M}_{-}^{\text {eff }}(\mathbb{C}, \mathbb{Q})$ that we denote by $\mathbf{M}(X)$. We will also use the compactly supported motive $\mathbf{M}^{c}(X)$ (see $\mathbf{M V W 0 6}$, Definition 16.13]). We write $\mathbf{D M}_{g m}(\mathbb{C}, R)$ for Voevodsky's derived category of geometric motives (see $\mathbf{M V W 0 6}$, p. 114]); this category can be most quickly described as follows: take the category $\mathbf{D} \mathbf{M}_{-}^{\mathrm{eff}}(\mathbb{C}, \mathbb{Z})$, invert the operation of tensoring with the Tate motive, and take the subcategory of compact objects in the resulting category.

\section{Rationality results for $\mathbb{Q}$-acyclic surfaces}

Fujita's structure theory for open algebraic surfaces [Fuj79] imposes strong topological restrictions on compactifications of an acyclic surface and, in particular, his results establish rationality of $\mathbb{Q}$-acyclic surfaces of logarithmic Kodaira dimension $\leqslant 0$. Gurjar, Shastri and Pradeep pushed this significantly further by treating the cases of acyclic surfaces of logarithmic Kodaira dimension 1 or 2 . The proof of Theorem 1 will rely on the difficult result, due to (subsets of) Gurjar, Shastri and Pradeep GP99, GPS97, GS89a, GS89b, PS97, that $\mathbb{Q}$-acyclic smooth complex surfaces are rational. The next statement recalls these results in the form in which they will be applied.

Theorem 2 (Fujita, Gurjar, Pradeep, Shastri). If $X$ is a $\mathbb{Z}$-acyclic (resp. $\mathbb{Q}$-acyclic) smooth complex surface, then $X$ is rational and affine. Furthermore, there exist a smooth proper surface $\bar{X}$ and an open immersion $j: X \hookrightarrow \bar{X}$ having the following properties:

(i) The variety $\bar{X}$ is a smooth, projective, rational surface, the boundary $\bar{X} \backslash X$ is a simple normal crossings divisor, and each irreducible component of $\bar{X}$ is a rational curve.

(ii) The group $\operatorname{Pic}(\bar{X})$ (resp. $\left.\operatorname{Pic}(\bar{X}) \otimes_{\mathbb{Z}} \mathbb{Q}\right)$ is freely generated by the irreducible components of $\bar{X} \backslash X$, and the intersection graph of these divisors forms a tree.

Proof. For the statements in (i), with the exception of the assertion of rationality, we proceed as follows. By resolution of singularities, we may always choose a smooth compactification $\bar{X}$ of $X$ whose boundary $\partial \bar{X}=\bar{X} \backslash X$ is a simple normal crossings divisor, in particular, all its irreducible components are smooth. To simplify notation, 
in the remainder of this proof we abuse notation by writing $X$ for $X(\mathbb{C})$ and $\partial \bar{X}$ for $\partial \bar{X}(\mathbb{C})$.

By either the assumption that $X$ is $\mathbb{Z}$-acyclic or $\mathbb{Q}$-acyclic, we can conclude by $\mathbf{F u j 8 2}$, Corollary 2.5] that $X$ is affine, $\mathbf{G}_{m}(X)=\mathbb{C}^{*}, \operatorname{Pic}(X)$ is a torsion group, $\bar{X}$ has geometric genus and irregularity 0 and the first Betti number of $\partial \bar{X}$ is zero. To check that $\partial \bar{X}$ has irreducible components consisting of smooth rational curves, it suffices to check that the boundary $\partial \bar{X}$ is both connected and simply connected. By Lefschetz duality we know that

$$
H^{i}(\bar{X}, \partial \bar{X}) \cong H_{4-i}(X), \text { and } H_{i}(\bar{X}, \partial \bar{X}) \cong H^{4-i}(X),
$$

using $\mathbb{Z}$-coefficients if $X$ is assumed $\mathbb{Z}$-acyclic and $\mathbb{Q}$-coefficients if $X$ is assumed $\mathbb{Q}$-acyclic. By the universal coefficient theorem, we deduce that $H^{i}(X, \mathbb{Z})$ (resp. $\left.H^{i}(X, \mathbb{Q})\right)$ vanishes for $1 \leqslant i \leqslant 4$. Now, the usual exact sequences for homology of a pair give rise to isomorphisms $H_{i}(\partial \bar{X}) \stackrel{\sim}{\rightarrow} H_{i}(\bar{X})$. One concludes that $\partial \bar{X}$ is connected. Now, we already know that the first Betti number of $\partial \bar{X}$ is 0 , and therefore [Fuj82, Theorem 2.8] (due to C.P. Ramanujam) allows us to conclude that $\partial \bar{X}$ is simply connected and, furthermore, a tree of rational curves.

For (ii), by [Fuj82, Corollary 2.9], we can conclude that $\operatorname{Pic}(X) \cong H^{2}(X, \mathbb{Z})$ and, thus, is trivial if $X$ is $\mathbb{Z}$-acyclic or torsion if $X$ is $\mathbb{Q}$-acyclic. By Hodge theory and Poincare duality one knows that $\operatorname{Pic}(\bar{X})$ is isomorphic to $H_{2}(\bar{X})$. The isomorphism $H_{2}(\partial \bar{X}) \stackrel{\sim}{\rightarrow} H_{2}(\bar{X})$ then allows one to conclude that $\operatorname{Pic}(\bar{X})\left(\right.$ resp. $\operatorname{Pic}(\bar{X}) \otimes_{\mathbb{Z}} \mathbb{Q}$ ) is generated by the irreducible components of the boundary divisor.

As mentioned before the proof, the difficult part of the statement is the assertion that $\bar{X}$ is rational. In that case, Gurjar-Pradeep prove [GP99, p. 259], that any $\mathbb{Q}$-acyclic variety is rational (since any $\mathbb{Z}$-acyclic variety is $\mathbb{Q}$-acyclic, this implies rationality in the $\mathbb{Z}$-acyclic case as well); this theorem builds on [GPS97, PS97]. For historical reasons, let us mention that the $\mathbb{Z}$-acyclic case was treated earlier in the papers GS89a, GS89b; see GS89b, Theorem 8.1], taking $X$ and $D$ in the statement of Gurjar-Shastri's theorem to be $\bar{X}$ and $\partial \bar{X}=\bar{X} \backslash X$ respectively (since we saw above that $X$ has geometric genus 0 ).

\section{Motives of acyclic surfaces}

Proof of Theorem $\mathbb{Q}$. We give the proof in the $\mathbb{Z}$-acyclic case; in the $\mathbb{Q}$-acyclic case the proof is identical after replacing $\mathbb{Z}$ by $\mathbb{Q}$ throughout. Fix a topologically $\mathbb{Z}$-acyclic smooth complex surface $X$, let $\bar{X}$ be a smooth compactification as described by Theorem 2, and write $\partial \bar{X}=\bar{X} \backslash X$.

The localization sequence for compactly supported motives (see MVW06, Theorem 16.15]) in our setup gives a triangle of the form:

$$
\mathbf{M}^{c}(\partial \bar{X}) \longrightarrow \mathbf{M}^{c}(\bar{X}) \longrightarrow \mathbf{M}^{c}(X) \longrightarrow \mathbf{M}^{c}(\partial \bar{X})[1] .
$$

Since both $\bar{X}$ and $\partial \bar{X}$ are projective, it follows from loc. cit. that $\mathbf{M}^{c}(\bar{X}) \cong \mathbf{M}(\bar{X})$ and $\mathbf{M}^{c}(\partial \bar{X}) \cong \mathbf{M}(\partial \bar{X})$. We will now describe the motive of the compactification $\bar{X}$ and the boundary $\partial \bar{X}$.

Since $\bar{X}$ is a smooth projective rational surface, we know that it is isomorphic to an iterated blow-up of either a Hirzebruch surface or projective space. Thus, we can compute its motive using the projective bundle formula (see, e.g., ibid., Theorem 15.12) and the blow-up triangle (see, e.g., ibid., 14.5.4). Consider two base cases: 
either $\bar{X}=\mathbb{P}^{2}$, in which case $\mathbf{M}\left(\mathbb{P}^{2}\right) \cong \oplus_{i=0}^{2} \mathbb{Z}(i)[2 i]$, or $\bar{X}=\mathbb{F}_{a}=\mathbb{P}\left(\mathcal{O}_{\mathbb{P}^{1}} \oplus \mathcal{O}_{\mathbb{P}^{1}}(a)\right)$, in which case $\mathbf{M}\left(\mathbb{F}_{a}\right)=\mathbb{Z} \oplus \mathbb{Z}(1)[2]^{\oplus 2} \oplus \mathbb{Z}(2)[4]$ by the projective bundle formula. If $Y$ is a smooth projective surface, then the blow-up distinguished triangle reduces to the identity

$$
\mathbf{M}\left(\mathrm{BI}_{y}(Y)\right) \cong \mathbf{M}(Y) \oplus \mathbb{Z}(1)[2],
$$

where $y \in Y(\mathbb{C})$. Thus, if $\bar{X}$ is an iterated blow-up of a rational surface, it follows that

$$
\mathbf{M}(\bar{X}) \cong \mathbb{Z} \oplus \mathbb{Z}(1)[2]^{\oplus r} \oplus \mathbb{Z}(2)[4]
$$

for some integer $r$.

Next, let us compute the motive of the boundary $\partial \bar{X}$; this boundary is a projective curve. Recall that an abstract blow-up of a variety $Y$ with center $Z$ is a proper morphism $p: Y^{\prime} \rightarrow Y$ that induces an isomorphism $\left(Y^{\prime}-Z^{\prime}\right)_{\text {red }} \cong(X-Z)_{\text {red }}$, where $Z^{\prime}=Z \times_{Y} Y^{\prime}$. Thus, any (partial) resolution of $\partial \bar{X}$ gives rise to an abstract blow-up. We know that $\partial \bar{X}$ is connected and the number of irreducible components of $\partial \bar{X}$ is equal to the rank $r$ of the Picard group of $\bar{X}$. We claim that we have a decomposition of the form

$$
\mathbf{M}(\partial \bar{X}) \cong \mathbb{Z} \oplus \mathbb{Z}(1)[2]^{\oplus r} .
$$

This is seen by induction on the number of connected components as follows.

By MVW06, Theorem 13.26], we have a triangle associated with any abstract blow-up:

$$
\mathbf{M}\left(Z^{\prime}\right) \longrightarrow \mathbf{M}\left(Y^{\prime}\right) \oplus \mathbf{M}(Z) \longrightarrow \mathbf{M}(Y) \longrightarrow \mathbf{M}\left(Z^{\prime}\right)[1]
$$

If $\partial \bar{X}$ has a single irreducible component, then this irreducible component is isomorphic to $\mathbb{P}^{1}$, and the result follows by the projective bundle formula above. Thus, assume that $\partial \bar{X}$ has two irreducible components. In this case, the resolution of $\partial \bar{X}$ has two connected components and the above triangle gives the required isomorphism. In general, there are a sequence of abstract blow-ups

$$
Y_{r-1} \longrightarrow Y_{r-2} \longrightarrow \cdots Y_{1} \longrightarrow \partial \bar{X}
$$

such that at each stage $Y_{i}$ has exactly one more connected component than $Y_{i-1}$ (blow up the intersection points in $\bar{X})$. Since each additional component is a smooth rational curve, it follows that its motive is isomorphic to $\mathbb{Z} \oplus \mathbb{Z}(1)[2]$ again by the projective bundle formula. Thus, $\mathbf{M}\left(Y_{r}\right) \cong \mathbb{Z}^{\oplus r} \oplus \mathbb{Z}(1)[2]^{\oplus r}$, and the morphism $Y_{i} \rightarrow Y_{i-1}$ thus kills a copy of $\mathbb{Z}$ at each stage. In particular, $\mathbf{M}(\partial \bar{X}) \cong \mathbb{Z} \oplus \mathbb{Z}(1)[2]^{\oplus r}$.

Combining the discussion of the previous two paragraphs, we see that the compactly supported motive of $X$ fits into a triangle of the form

$$
\mathbb{Z} \oplus \mathbb{Z}(1)[2]^{\oplus r} \longrightarrow \mathbb{Z} \oplus \mathbb{Z}(1)[2]^{\oplus r} \oplus \mathbb{Z}(2)[4] \longrightarrow \mathbf{M}^{c}(X) \longrightarrow \mathbb{Z}[1] \oplus \mathbb{Z}(1)[3]^{\oplus r}
$$

Since both $\bar{X}$ and $\partial \bar{X}$ are connected, the irreducible components of $\partial \bar{X}$ generate the Picard group of $\bar{X}$, and in the localization sequence, the first map of the triangle is defined by the push-forward of cycles. It follows that the first map is split injective and thus has cokernel exactly $\mathbb{Z}(2)[4]$. Thus, the map $\mathbb{Z}(2)[4] \rightarrow \mathbf{M}^{c}(X)$ is an isomorphism in $\mathbf{D M}^{-}(\mathbb{C}, \mathbb{Z})$. 
To obtain the statement about geometric motives, we use duality theory. The morphism $\mathbb{Z}(2)[4] \rightarrow \mathbf{M}^{c}(X)$ is induced by "flat pullback" from the structure morphism $X \rightarrow \operatorname{Spec} \mathbb{C}$ by $\mathbf{\text { Voe00, }}$, Corollary 4.2.4]. The statement about geometric motives then follows immediately from [MVW06, Example 20.11] or [Voe00, Theorem 4.3.7.3].

\section{Complements on motives of $\mathbb{Z}$-acyclic varieties}

There are several methods for producing new $\mathbb{Z}$-acyclic smooth complex varieties from a given one; here we review a procedure using affine modifications in the sense of Zariski. Suppose $X \supset D \supset C$ is a triple consisting of a smooth quasi-projective variety $X$, an irreducible hypersurface $D \subset X$, and a smooth subvariety $C \subset D$ everywhere of codimension $d \geqslant 2$ in $X$. Let $\mathrm{Bl}_{C}(X)$ be the blow-up of $X$ along $C$. Let $D^{\prime} \subset \mathrm{Bl}_{C}(X)$ be the proper transform of $D$. The Kaliman modification of $X$ along $D$ with center $C$ is defined by $\mathrm{Bl}_{C, D}(X):=\mathrm{Bl}_{C}(X) \backslash D^{\prime}$ (cf. [Zaי99, Definition 4.1]). Also, let $E^{\prime}$ denote the intersection of the exceptional divisor of the blow-up $\mathrm{Bl}_{C}(X) \rightarrow X$ with $\mathrm{Bl}_{C, D}(X)$. The following result summarizes the basic properties of Kaliman modifications.

Proposition 3 (See, e.g., Zaי̃99, Lemmas 4.1 and 4.2]). Suppose $X \supset D \supset C$ is a triple as above and consider the Kaliman modification $\sigma_{C, D}: \mathrm{Bl}_{C, D}(X) \rightarrow X$. Assume for simplicity that $D$ is smooth.

(i) The morphism $\sigma_{C, D}$ induces an isomorphism $\mathrm{Bl}_{C, D}(X) \backslash E^{\prime} \rightarrow X \backslash D$.

(ii) The restricted morphism $\left.\sigma_{C, D}\right|_{E^{\prime}}: E^{\prime} \rightarrow C$ is a Zariski locally trivial bundle with affine space fibers.

(iii) The morphism $\pi_{1}\left(\mathrm{Bl}_{C, D}(X)\right) \rightarrow \pi_{1}(X)$ is an isomorphism.

(iv) If $C$ and $D$ are $\mathbb{Z}$-acyclic, then $\mathrm{Bl}_{C, D}(X)$ is $\mathbb{Z}$-acyclic if and only if the same holds for $X$.

Similar statements can be made for Kaliman modifications of $\mathbb{Q}$-acyclic varieties. The next result follows easily by combining Proposition 3 with the existence and functoriality of localization triangles for motives with compact support ( MVW06, Theorem 16.25]), the triangulated category version of the 5-lemma, and the motivic Thom isomorphism (see, e.g., [MVW06, Theorem 15.15]).

Proposition 4. Suppose $\varphi: \mathrm{Bl}_{C, D}(X) \rightarrow X$ is a Kaliman modification, and let $E^{\prime}$ be the exceptional divisor of $\varphi$. Then assuming $C$ and $D$ are smooth, $\mathbf{M}(\varphi)$ is an isomorphism if and only if the map $C \hookrightarrow D$ induces an isomorphism $\mathbf{M}(C) \rightarrow \mathbf{M}(D)$. If, furthermore, $\mathbf{M}(C) \rightarrow \mathbf{M}(D)$ is an isomorphism and $\mathbf{M}(X) \cong \mathbb{Z}$ it follows that $\mathbf{M}\left(X^{\prime}\right) \cong \mathbb{Z}$ as objects in $\mathbf{D M}_{\mathbb{Z}}(\mathbb{C})$.

Example 5. Consider $\mathbb{A}^{2}$ with coordinates $x$ and $y$. Let $V_{n, m}$ be the variety constructed in the following manner: blow up $\mathbb{A}^{2}$ at the point $(1,1)$, and remove the proper transform of the curve $\Gamma_{n, m}=\left\{x^{n}-y^{m}=0\right\}$ (where $n>m>1$ are coprime natural numbers). We write $L_{n, m}$ for the affine line corresponding to the complement of the intersection of the exceptional divisor of the blow-up and the curve $\Gamma_{n, m}$. The resulting surface, called a tom Dieck-Petrie surface, is a topologically contractible smooth surface of logarithmic Kodaira dimension 1 (see tDP90 for the 
general theory of such surfaces). More explicitly, the surfaces $V_{n, m}$ can be described as hypersurfaces in $\mathbb{A}^{3}$ (see tDP90, Theorem $\mathrm{C}$ and $\left.\S 6\right]$ ) defined by the equations

$$
\frac{(x z+1)^{m}-(y z+1)^{n}}{z}=1 \text {. }
$$

Performing repeated affine modifications on surfaces of this form allows one to produce positive dimensional families of non-isomorphic $\mathbb{Z}$-acyclic varieties; see Zaי̌99. Theorem 2.6(d)] for more details.

Example 6. It is easy to construct situations satisfying the hypotheses of the previous proposition. For example, take any $\mathbb{Z}$-acyclic smooth surface $X$ not isomorphic to $\mathbb{A}^{2}$ and consider the variety $X \times \mathbb{A}^{n}$ for some integer $n>0$. Fix a point $x \in X$ (or a contractible smooth curve) and consider a hypersurface of the form $X \times \mathbb{A}^{n-1}$; this is a $\mathbb{Z}$-acyclic hypersurface. We can perform a Kaliman modification of $X \times \mathbb{A}^{n}$ along $X \times \mathbb{A}^{n-1}$ with center $x$ (or the contractible smooth curve) and, by combining Proposition 3 (iii) and Proposition 4 , obtain a $\mathbb{Z}$-acyclic smooth complex variety of dimension $n+2$ whose motive is isomorphic to $\mathbb{Z}$. In many cases, the resulting varieties are not isomorphic to affine space.

\section{References}

[Fuj79] T. Fujita, On Zariski problem, Proc. Japan Acad. Ser. A Math. Sci. 55 (1979), no. 3, 106-110.

[Fuj82] T. Fujita, On the topology of noncomplete algebraic surfaces, J. Fac. Sci. Univ. Tokyo Sect. IA Math. 29 (1982), no. 3, 503-566.

[GP99] R.V. Gurjar and C.R. Pradeep, $\mathbb{Q}$-homology planes are rational. III, Osaka J. Math. 36 (1999), no. 2, 259-335.

[GPS97] R.V. Gurjar, C.R. Pradeep, and A.R. Shastri, On rationality of logarithmic Q-homology planes. II, Osaka J. Math. 34 (1997), no. 3, 725-743.

[GS89a] R.V. Gurjar and A.R. Shastri, On the rationality of complex homology 2-cells. I, J. Math. Soc. Japan 41 (1989), no. 1, 37-56.

[GS89b] R.V. Gurjar and A.R. Shastri, On the rationality of complex homology 2-cells. II, J. Math. Soc. Japan 41 (1989), no. 2, 175-212.

[Hub08] A. Huber, Slice filtration on motives and the Hodge conjecture (with an appendix by J. Ayoub), Math. Nachr. 281 (2008), no. 12, 1764-1776.

[MVW06] C. Mazza, V. Voevodsky, and C. Weibel, Lecture notes on motivic cohomology, Clay Mathematics Monographs 2, American Mathematical Society, Providence, RI, 2006.

[PS97] C.R. Pradeep and A.R. Shastri, On rationality of logarithmic Qhomology planes. I, Osaka J. Math. 34 (1997), no. 2, 429-456.

[tDP90] T. tom Dieck and T. Petrie, Contractible affine surfaces of Kodaira dimension one, Japan. J. Math. (N.S.) 16 (1990), no. 1, 147-169.

[Voe00] V. Voevodsky, Triangulated categories of motives over a field, in Cycles, transfers, and motivic homology theories, Ann. of Math. Stud. 143, pages 188-238. Princeton Univ. Press, Princeton, NJ, 2000. 
[Zaי̌99] M. Zaŭdenberg, Exotic algebraic structures on affine spaces, Algebra $i$ Analiz 11 (1999), no. 5, 3-73, translation in St. Petersburg Math. J. 11 (2000), no. 5, 703-760.

Aravind Asok asok@usc.edu

Department of Mathematics, University of Southern California, 3620 S. Vermont Ave. KAP 104, Los Angeles, CA 90089-2532, United States 